\title{
The management of febrile infants 30-90 days old without source hospitalised in Crete, Greece, 2008-2017: a single centre experience
}

Varanaki Maria Eleni, Kalaitzidou loanna, Ladomenou Fani, Vlachaki Georgia Department of Paediatrics, Venizeleion General Hospital, Crete, Greece

\section{Background:}

"Fever without source" (FWS) is a common diagnostic challenge in infancy. However, few infants with FWS have a serious bacterial infection (SBI) and most non-toxic appearing can be managed using low risk laboratory and clinical criteria.

\section{Aim:}

To analyze our management approach of FWS in infancy over the ten-year study period.

\section{Population and Methods:}

\section{Place}

- Paediatric Department, Venizeleion General Hospital, Heraklion, Crete, Greece
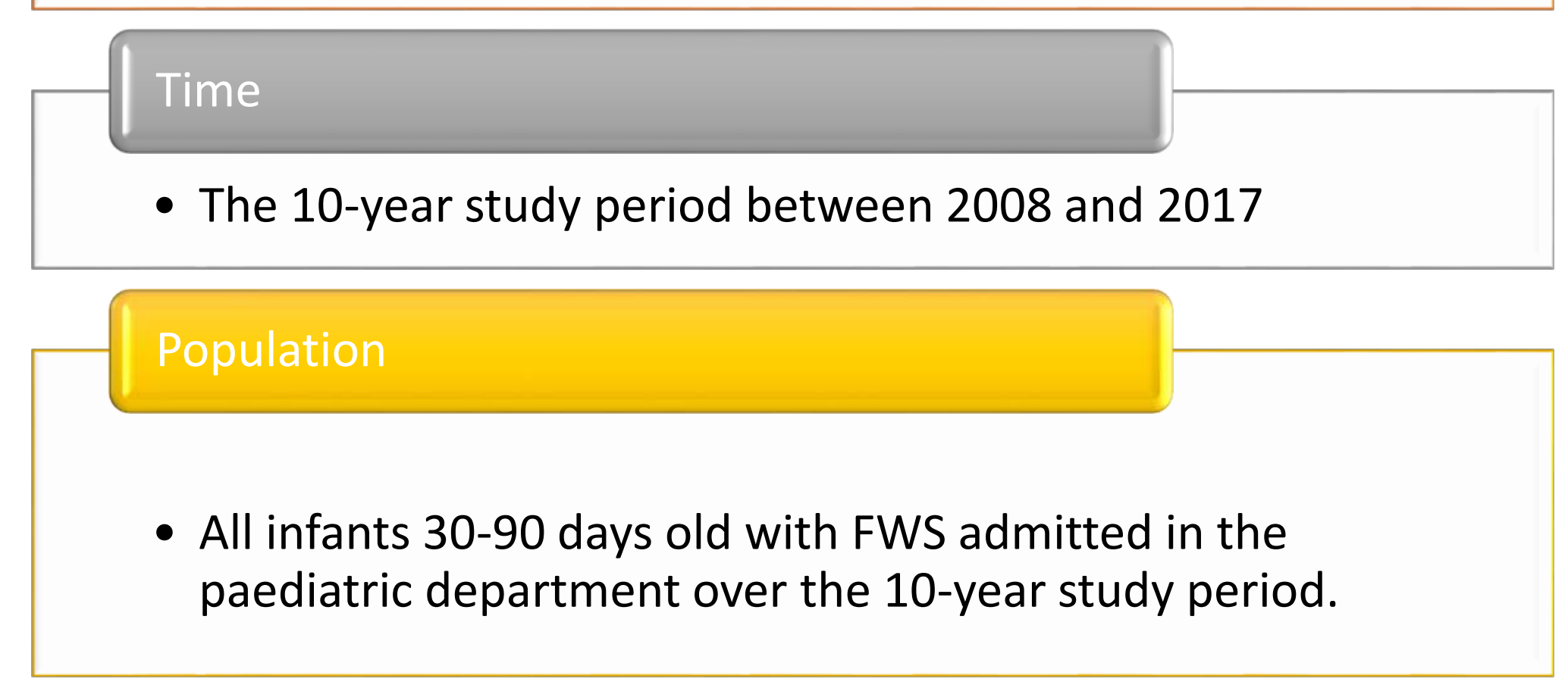

\section{Methods}

- Retrospective registry-based cohort study of the management of all participants during the study period

- The infants considered as low risk for serious bacterial infection (SBI) were those fulfilling the 'Rochester criteria'

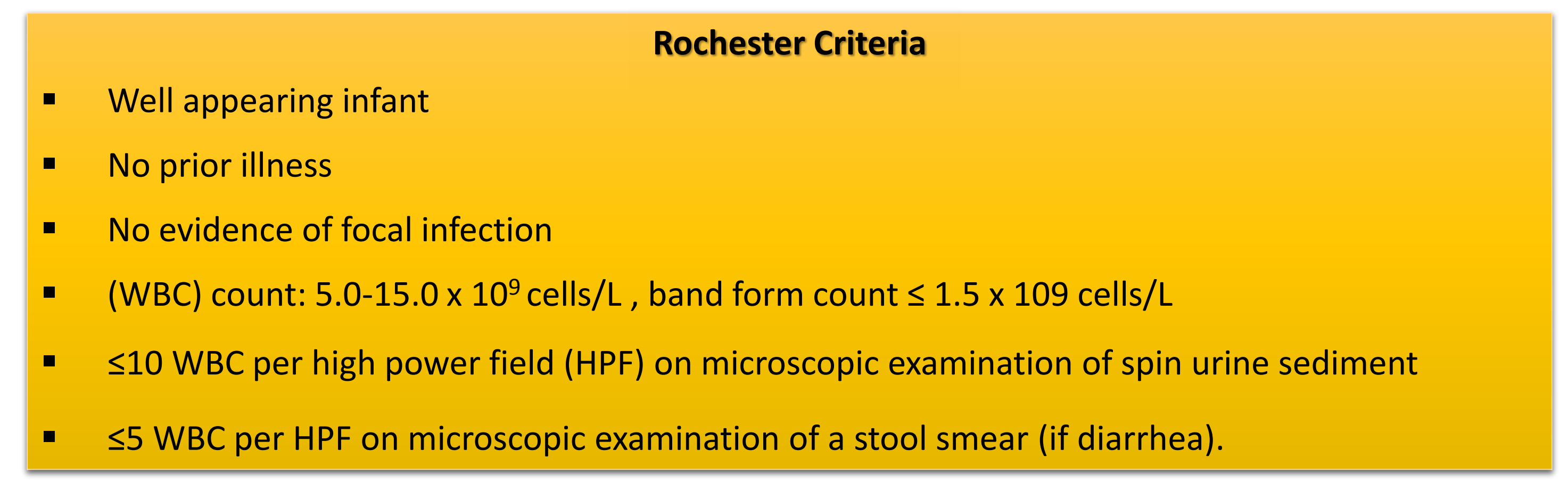

Results:

Fulfillment of Rochester Criteria

- 219 infants were enrolled in the study ( $46.6 \%$ male).

- 145/219 (66.2\%) met all low risk criteria.

- 193/219 (88.1\%) received intravenous antibiotics.

- $84,8 \%$ of infants who met the low risk criteria received intravenous antibiotics.

- Third generation cephalosporins were the most frequently administered antibiotics (98.4\%).

- $93.8 \%$ of infants who met the criteria underwent complete sepsis evaluation, including a lumbar puncture.

- The management decision was not significantly affected by the gender, age, previous NICU admission or vaccination status.

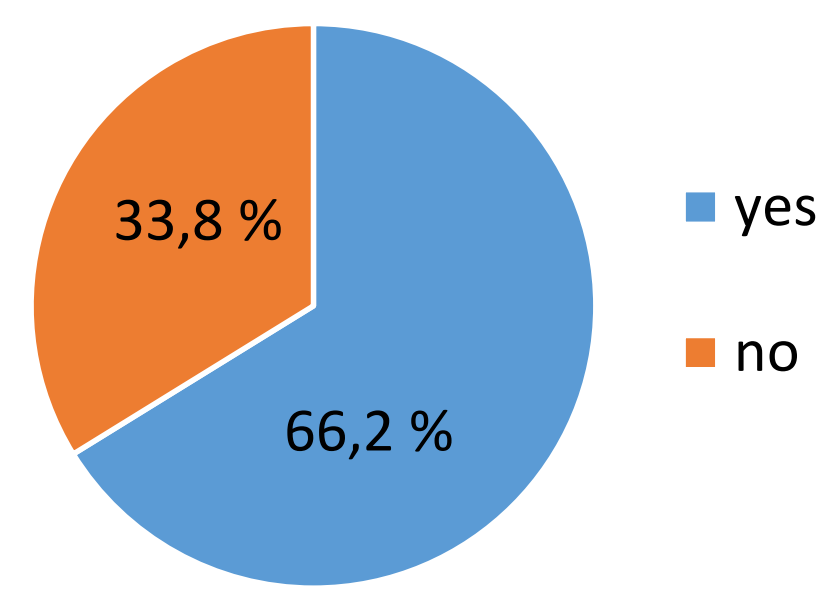

Management of infants who met the Criteria

\section{Conclusions:}

- Received antibiotics $\quad$ Did not receive antibiotics

Our results reveal the need to reinforce medical education in order to avoid inappropriate use of antibiotics in low risk infants with FWS, given that antibiotic resistance is a growing public health concern worldwide.

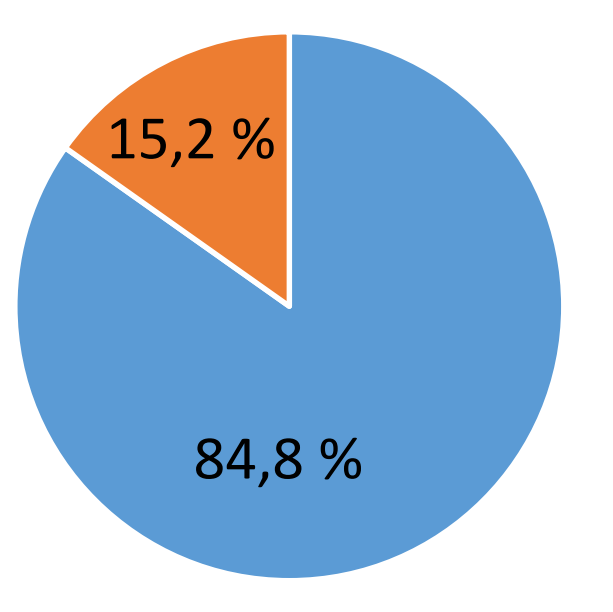

